\title{
Deglutição no envelhecimento e a odontologia
}

Swallowing in aging and dentistry

\author{
Rafaela Soares Rech* \\ Bárbara Niegia Garcia de Goulart* \\ Alexandre Baumgarten ${ }^{* * *}$ \\ Juliana Balbinot Hilgert ${ }^{* * * * *}$
}

\section{Resumo}

As alterações morfológicas e funcionais que atingem o sistema estomatognático podem ser agravadas por perdas de dentes e utilização de prótese dentária, que comprometem o ato mastigatório e a deglutição de idosos. Objetivo: assim, propõe-se a presente revisão de literatura, que tem como objetivo estreitar os conhecimentos que devem existir entre a Fonoaudiologia e a Odontologia, discorrendo sobre as evidências atuais e histórico-conceituais mais relevantes sobre deglutição que aproximam as duas especialidades, principalmente em indivíduos idosos. Revisão de literatura: foram incluídos no estudo 101 trabalhos, selecionados a partir das bases de dados PubMed Central, Scientific Eletronic Library Online, Science Direct, SciELO, Periódico Capes e identificados até abril de 2018 em inglês, português e espanhol. Destaca-se entre os achados a crescente inserção da Odontologia na atuação da disfagia orofaríngea. A falta de cuidados coordenados na atenção ao idoso interfere no seu bem-estar geral e na sua qualidade de vida. Considerações finais: as evidências da literatura demonstram importante avanço na identificação, no diagnóstico e na reabilitação da disfagia orofaríngea, que, por meio da reabilitação bucal, demonstrou ser relevante na prevenção de alterações na deglutição.

Palavras-chave: Deglutição. Disfagia. Envelhecimento. Fonoaudiologia. Odontologia. Therapy. Swallowing.

\section{Introdução}

Ao examinar brevemente a história da Odontologia, observa-se que, em 1927, Rogers ${ }^{1}$ (1927), cirurgião-dentista norte-americano, publicou casos de mordida aberta abarcando problemas de deglutição. Em 1946, Rix ${ }^{2}$ (1946), cirurgião-dentista inglês, escreveu sobre a deglutição e a posição dos dentes. Em 1951, Straub ${ }^{3}$ (1951), cirurgião-dentista norte-americano, escreveu sobre a etiologia do hábito inadequado de deglutir. A partir da década de 1950, surgiram os fonoaudiólogos, atuando na área da motricidade orofacial, prosseguindo com os avanços da Odontologia, assumindo os trabalhos de avaliação e reabilitação dos problemas miofuncionais orofaciais ${ }^{4}$. Em 1971, publicou-se o primeiro livro com foco multidisciplinar, para cirurgiões-dentistas e fonoaudiólogos, que abordava hábitos anormais da deglutição, diagnóstico e reabilitação ${ }^{5}$. Doravante, estreitaram-se os elos entre esses profissionais, que tratam da função da alimentação ao mesmo tempo em que estudam essa função, que desempenha um papel vital no cotidiano de todos os indivíduos.

A precária saúde bucal entre os idosos representa um importante problema de saúde pública ${ }^{6}$. O impacto negativo das precárias condições bucais sobre a vida cotidiana é particularmente significante entre os indivíduos com perda de dentes ${ }^{7}$. A

Mestre em Saúde Bucal Coletiva pelo Programa de Pós-Graduação em Odontologia, Faculdade de Odontologia, Universidade Federal do Rio Grande do Sul, Porto Alegre, Rio Grande do Sul, Brasil.

* Doutora, professora do Programa de Pós-Graduação em Epidemiologia, Faculdade de Medicina e Departamento de Saúde e Comunicação Humana, Faculdade de Psicologia, Universidade Federal do Rio Grande do Sul, Porto Alegre, Rio Grande do Sul, Brasil.

*** Mestre em Epidemiologia pelo Programa de Pós-Graduação em Epidemiologia, Faculdade de Medicina, Universidade Federal do Rio Grande do Sul, Porto Alegre, Rio Grande do Sul, Brasil.

**** Doutora, professora do Programa de Pós-Graduação em Odontologia, Faculdade de Odontologia, e do Programa de Pós-Graduação em Epidemiologia, Faculdade de Medicina e Departamento de Odontologia Preventiva e Social, Faculdade de Odontologia, Universidade Federal do Rio Grande do Sul, Porto Alegre, Rio Grande do Sul, Brasil. 
saúde bucal não pode ser vista separadamente da saúde geral. Desse modo, a comunidade científica tem demonstrado cada vez mais interesse em possíveis relações entre as condições orais e os desfechos sistêmicos ${ }^{8}$.

A nova definição universal de saúde bucal aprovada pela World Dental Federation General Assembly (FDI) ressalta a importância de analisar a integralidade da saúde bucal com a saúde geral dos indivíduos, levando em consideração seus valores, percepções e expectativas. Além disso, confirma que as habilidades de mastigação e de deglutição são importantes funções a serem investigadas na saúde bucal e devem representar importante prática clínica dos cuidados odontológicos ${ }^{9}$.

Assim, a presente revisão de literatura objetiva estreitar os conhecimentos que devem existir entre a Fonoaudiologia e a Odontologia, discorrendo sobre as evidências atuais e histórico-conceituais que aproximam as duas especialidades, principalmente em indivíduos idosos.

\section{Materiais e método}

O desenvolvimento desta revisão de literatura seguiu os preceitos do estudo exploratório, por meio de uma pesquisa bibliográfica em artigos e livros científicos sobre o tema. Foi realizada uma pesquisa nas bases de dados PubMed Central, Scientific Eletronic Library Online, Science Direct, SciELO, Periódico Capes, que contou com estudos identificados até abril de 2018, utilizando os descritores em português e sua correspondência em inglês, associados pelos operadores booleanos AND e OR: dentistry, deglutition, deglutition disorders, oral health, mastication, orofacial motricity, speech-language therapist e aged. Restringiu-se a busca para textos publicados em português, inglês e espanhol e disponíveis na íntegra. Artigos relevantes sobre o tema foram selecionados, e, a partir das referências deles, outras publicações foram localizadas.

Para a coleta das informações, foram realizadas a leitura exploratória de todo o material selecionado, verificando incialmente se a publicação era de interesse para o estudo; e a leitura seletiva dos artigos, na qual se aprofundaram as partes de interesse para a revisão. Na etapa seguinte, realizou-se o registro das informações extraídas das fontes em instrumento específico (autores, ano, método, resultados e conclusões). A análise e a interpretação dos resultados foram realizadas por meio de uma leitura analítica e crítica, com a finalidade de selecionar estudos adequados metodologicamente, que contribuíssem para a construção do presente estudo.

A apresentação dos resultados (revisão de literatura) e a discussão dos dados foram realizadas de forma descritiva, possibilitando ao leitor a avaliação da aplicabilidade da revisão elaborada, visando a impactar positivamente na interdisciplinaridade das ciências (Odontologia e Fonoaudiologia) e na prática clínica dos profissionais dessas áreas.

\section{Revisão da literatura}

\section{Deglutição e o processo do envelhecimento}

A deglutição é um mecanismo neurofisiológico, semiautomático, complexo, contínuo, mediado por núcleos do tronco cerebral ${ }^{10}$. A função de deglutir desempenha um papel vital no cotidiano de todos os indivíduos ao transportar nutrientes e energia necessários da cavidade oral ao estômago, garantindo que nenhuma substância vá para a via aérea ${ }^{11}$.

O processo de envelhecimento pode ser acompanhado pelo declínio de capacidades tanto físicas como cognitivas dos idosos, de acordo com suas características de vida e suas heranças genéticas ${ }^{12}$. A deglutição, por ser um mecanismo complexo, que envolve os sistemas - neurológico, respiratório e digestivo -, pode sofrer acentuadas alterações decorrentes do envelhecimento, ocasionando distúrbios em uma ou mais fases de seu processo ${ }^{13}$.

Para designar as modificações normais no processo de deglutição decorrentes da degeneração fisiológica em virtude do envelhecimento sadio das fibras nervosas e musculares e dos componentes anatômicos craniofaciais, usa-se a denominação presbifagia. Entretanto, as alterações sofridas estão diretamente associadas à maior chance de fragilização, bem como ao desenvolvimento de disfagia ${ }^{14}$. As principais alterações se relacionam a: lentificação na manipulação do alimento, escape anterior devido à dificuldade de vedamento labial, atraso na ejeção do bolo alimentar devido à diminuição de força da língua e diminuição dos movimentos peristálticos e da abertura dos esfíncteres ${ }^{15,16}$.

Nos idosos, ocorre o enfraquecimento da coordenação entre as fases da deglutição. Em jovens, as fases estão intimamente ligadas e muitas vezes se sobrepõem, atuando de forma protetiva ${ }^{17}$. Por meio da videofluoroscopia da deglutição, podem-se observar atraso de 0,4 segundos para o estímulo da fase faríngea, diminuição do tempo de duração total do reflexo faríngeo e diminuição da duração de abertura cricofaríngea para 0,25 segundos. O atraso do disparo do reflexo faríngeo, muitas vezes, resulta em acúmulo do bolo alimentar nas valéculas, condição que atrasa a elevação faríngea e consequentes processos subsequentes da deglutição ${ }^{18}$. As reduções da massa muscular e da elasticidade do tecido conjuntivo podem afetar negativamente o fluxo do bolo alimentar no processo da deglutição, dificultando seu deslizamento craniocaudal e desestabilizando a ligação entre os processos ${ }^{19}$.

A fase oral da deglutição compreende a mastigação, que depende de um complexo integrado por músculos, ligamentos, estruturas ósseas e dentes, 
controlado pelo sistema nervoso central. Os músculos envolvidos na mastigação realizam movimentos e posturas que ora aproximam, ora afastam os dentes, ou exacerbam a pressão interoclusal. A mandíbula desenvolve os movimentos de elevação, abaixamento, protrusão, retração e lateralização, influenciados pelos músculos responsáveis pela mastigação (masseter, temporal anterior, pterigoideos medial e lateral, digástrico, estilo-hioideo, milo-hioideo e genio-hioideo). A eficiência da mastigação é garantida pela força exercida na contração desses músculos, e, para que a deglutição se processe de maneira normal, faz-se necessário o equilíbrio entre os músculos periorais, mastigadores e língua ${ }^{20}$. Entretanto, no envelhecimento, sabe-se que esses processos acontecem de forma prejudicada, pois a capacidade muscular comprometida diminui a rapidez, não há muita força e percepção ${ }^{16}$.

As alterações morfológicas e funcionais que atingem o sistema estomatognático podem ser agravadas por perdas de dentes e pela utilização de prótese dentária, que compromete o ato mastigatório ${ }^{21}$. As ausências estão associadas à seleção da consistência alimentar, à dificuldade na deterioração do alimento e à capacidade mastigatória. No indivíduo total ou parcialmente edentado, há uma dificuldade intrínseca no trabalho do bolo alimentar, o que o obriga a procurar alimentos mais adequados à eficiência mastigatória ${ }^{15}$.

Além de apresentar uma mastigação prejudicada, o idoso tem dificuldade de controlar o bolo alimentar. Essa dificuldade está associada à capacidade muscular, que perde mobilidade, força e autopercepção, acrescida pela diminuição gustativa, por xerostomia e por alterações da mucosa oral. Um movimento posterior prematuro de ingestão do alimento compromete a integridade da via respiratória ${ }^{22}$. A ingestão de bolo alimentar em volume inadequado (demasiadamente grande), realizado rapidamente (com pouca trituração), em posição anormal é comum nos idosos ${ }^{23}$.

A xerostomia é outro fator prevalente em idosos, variando entre 20 e $60 \%{ }^{24,25}$. Destacam-se entre as suas associações os medicamentos utilizados e sua quantidade, entretanto, são relevantes também o hábito tabágico, a ingestão de água e os hábitos de higiene bucal dos indivíduos ${ }^{24,25}$. Entre os medicamentos, os diuréticos e os antidepressivos são os mais prejudiciais para a salivação ${ }^{24}$. Em investigação com 529 pacientes geriátricos, $72 \%$ relataram apresentar a boca seca em algum momento do dia, e $55 \%$ utilizavam medicamentos para evitar o ressecamento. Observou-se associação significativa com dificuldade de mastigação, formação inadequada do bolo alimentar e dificuldade para iniciar o processo de deglutição ${ }^{26}$. Tradicionalmente, o tratamento de xerostomia, quando associado à diminuição do fluxo salivar, tem sido realizado com medidas paliativas, como o uso de substitutivos salivares, que são, entretanto, facilmente removidos da boca durante a deglutição. Algumas terapias tópicas para a xerostomia podem ser: goma de mascar, gel lubrificante, colutórios, pastas de dentes, pastilhas estimulantes, spray de mucina, umidificadores e substitutos da saliva liberados por dispositivos intrabucais ${ }^{27}$.

Com o envelhecimento, ocorre a diminuição da massa muscular esquelética, imiscuindo-se nas atividades diárias dos idosos. Mantê-la é essencial para o desenvolvimento adequado de todas as funções físicas ${ }^{28}$. Declínios na massa e na força muscular são esperados; entretanto, há importante variabilidade entre as perdas de peso e as suas associaçõe ${ }^{29}$. A função orofaríngea, responsável por parte do processo da deglutição, é extremamente importante para a ingestão nutricional adequada e necessária para evitar a fragilidade e a sarcopenia. Alguns estudos têm demonstrado a relação entre as condições bucais e as nutricionais, tais como o número de dentes remanescentes, o suporte oclusal e o uso de próteses dentárias ${ }^{30}$.

A desnutrição, complicação da alteração no processo de deglutição, pode aumentar o risco de morbidade, acarretando aumento da fragilidade, da demanda sobre os serviços de saúde e, consequentemente, do sistema de saúde. Suas consequências são disparadoras do declínio geral da saúde dos idosos, aumentando o risco de mortalidade ${ }^{31}$.

\section{Disfagia}

A disfagia é definida como uma alteração na deglutição. Os sinais e sintomas da disfagia podem envolver boca, faringe, laringe e/ou esôfago ${ }^{32}$. Essa condição manifesta-se como disfagia orofaríngea (dificuldade ou incapacidade de formar ou mover o bolo alimentar com segurança desde a boca até o esôfago) ou como disfagia esofágica (dificuldade de o bolo alimentar chegar ao estômago a partir do esôfago adequadamente $)^{33}$. O resultado de ambas complicações é a penetração ou a aspiração alimentar ou do conteúdo gástrico no trato respiratório, sendo que a penetração é a passagem do alimento na laringe, mas não abaixo das pregas vocais verdadeiras; enquanto que a aspiração é a passagem que ultrapassa as pregas vocais verdadeiras e percorre a traqueia até a chegada no pulmão $0^{34}$. As principais consequências da disfagia são pneumonia aspirativa, desidratação, desnutrição e aumento da mortalidade $^{35}$. Cabe salientar que a disfagia orofaríngea é um campo em que atua a Fonoaudiologia e possibilita a interface com a Odontologia descrita nesta revisão.

Pode-se adquirir a disfagia a partir de diversas etiologias, incluindo doenças neurológicas, como demência, doença de Parkinson, esclerose múltipla e acidente vascular cerebral ${ }^{36}$. Câncer de cabeça e de pescoço, cirurgia da coluna cervical, traumatismo cranioencefálico, fibromialgia, artrite reumatoide e doença pulmonar obstrutiva crônica também estão associados ao seu desenvolvimento, assim como 
muitas síndromes genéticas ${ }^{37,38,39}$. Além disso, o envelhecimento ativo está associado à sua propensão $0^{40}$.

Estima-se que mais de 16 milhões de idosos estadunidenses, 30 milhões de idosos europeus e 10 milhões de idosos japoneses tenham disfagia ${ }^{41}$. Dados revelam que de $13 \%$ a $35 \%$ dos idosos independentes reportam sintomas disfágicos, sendo que a maioria não procura tratamento ${ }^{42}$. Além disso, um a cada nove idosos saudáveis autorrelata sinais de disfagia $^{43}$, e $40 \%$ dos idosos saudáveis, com ou sem queixas relativas à deglutição, relataram-na em algum momento da vida ${ }^{4}$.

A fisiopatologia das complicações associadas à disfagia orofaríngea funcional do idoso evidencia que: a disfagia, o ressecamento da mucosa orofaríngea e a colonização orofaríngea de bactérias gram-negativas ocasionam alterações na segurança e na eficiência da deglutição, que podem originar infecção respiratória, aspiração (pneumonia por aspiração), desnutrição (perda da massa muscular, alteração da imunidade e da cicatrização) e desidratação (hipovolemia, alterações renal e cardiovascular e diminuição do estado de consciência), procedendo um estado de fragilização, comprometendo a capacidade funcional, causando lesões de pele, imunossupressão, infecções intercorrentes e morbidade, todos integrados à possível mortalidade ${ }^{45,46}$.

Além de afetar negativamente a saúde física, a disfagia interfere na qualidade de vida. Indivíduos disfágicos referem efeito prejudicial na socialização, pois não são capazes de alimentar-se com a família e com os amigos, resultando em isolamento e depressão. Em entrevista com indivíduos disfágicos em quatro diferentes países europeus, $45 \%$ relataram experiências agradáveis ao se alimentar; entretanto, $36 \%$ revelaram não se alimentar conjuntamente com outras pessoas devido à disfagia, 37\% admitiram sentir envergonha por causa da disfagia, e $41 \%$ já experienciaram, ao menos uma vez, ansiedade e pânico devido à doença. Além disso, 55\% referiram apresentar uma vida menos agradável com a presença da disfagia. A disfagia afeta a dignidade, a autoestima e a rotina dos indivíduos, causando ansiedade e angústia na hora das refeições ${ }^{47}$.

\section{Repercussões da saúde bucal na disfagia}

No Japão, uma das causas mais comuns de morte em idosos é a pneumonia por aspiração. A manutenção da saúde bucal por cirurgiões-dentistas e a manutenção da alimentação por via oral são elementos importantes no cuidado aos pacientes disfágicos, especialmente nos casos de doenças neurológicas, cirurgias de cabeça e pescoço e em idosos, evitando a pneumonia aspirativa. Ressalta-se que, além da reabilitação fonoaudiológica e dos cuidados de saúde bucal, o tratamento dental é incluído aos cuidados clínicos durante as intervenções. Sendo assim, quando há necessidade, o paciente é atendido por uma equipe interdisciplinar especializada, que compreende médicos, cirurgiões-dentistas e fonoaudiólogos. As intervenções clínicas realizadas são: reabilitação funcional, manutenção da alimentação, assistência durante a refeição, cuidados de saúde bucal e tratamento odontológico ${ }^{48}$.

Os cuidados da saúde bucal são importantes na prevenção da pneumonia aspirativa em idosos ${ }^{49}$. O cuidado oral demonstra-se eficiente também na prevenção da pneumonia aspirativa em idosos totalmente dependentes de sonda. Um estudo teve como objetivo avaliar os efeitos do treinamento funcional e do cuidado bucal na frequência de pneumonia em idosos disfágicos com dieta alimentar exclusivamente por sonda. Os indivíduos foram divididos em dois grupos, sendo que um recebeu a intervenção funcional e os cuidados bucais por um grupo de saúde bucal treinado, e o outro recebeu apenas a intervenção funcional por um grupo não treinado, uma vez por semana, durante três anos. O grupo que recebeu as intervenções funcionais e de cuidados bucais diminuiu ano a ano a incidência de pneumonia, demonstrando-se uma prática importante a ser inserida nos cuidados dos disfágicos ${ }^{50}$.

Em Tóquio, cirurgiões-dentistas especialistas em disfagia avaliaram a massa muscular esquelética orofacial, o estado oclusal e a função da deglutição em idosos fragilizados com no mínimo 75 anos de idade. Foram avaliados também as funções física e cognitiva e o estado nutricional por meio de entrevistas com os cuidadores. $\mathrm{O}$ resultado principal do estudo demonstrou que a massa muscular esquelética orofacial foi associada ao estado nutricional. Os achados demonstram que os tratamentos dentários e o uso de próteses são importantes para manter a massa muscular esquelética, especialmente nos homens, favorecendo a deglutição e consequentemente a nutrição dos idosos ${ }^{51}$.

Um estudo demonstrou que a perda de oclusão dos dentes posteriores está associada ao risco de disfagia orofaríngea em idosos residentes de instituição de longa permanência. Seus achados revelam que a manutenção e a restauração da oclusão dos dentes posteriores podem ser uma medida efetiva para prevenir a disfagia ${ }^{52}$. Um estudo de coorte avaliou durante cinco anos 1988 idosos que viviam na comunidade. Durante o passar do tempo, indivíduos com menos dentes remanescentes (0-12 e 13-22) apresentaram maior propensão a desenvolver alterações na deglutição, quando comparados aos idosos com dentes remanescentes (27-32) ${ }^{53}$. Em consonância, um estudo demonstrou que a não funcionalidade oral está associada à maior prevalência de disfagia em idosos que vivem na comunidade ${ }^{54}$.

Sabe-se que as próteses mal ajustadas prejudicam a mastigação e interferem na deglutição. Em um estudo realizado com mulheres com idades entre 60 e 80 anos sem queixas ou dificuldades na deglutição, as perdas dentárias e as próteses mal 
adaptadas prejudicavam a fase preparatória oral da deglutição $0^{55}$

Recentemente, um estudo propôs uma avaliação simplificada da disfagia direcionada a cirurgiões-dentistas, para o reconhecimento de alterações na deglutição em idosos precocemente. O exame clínico simplificado da deglutição por cirurgiões-dentistas foi considerado um método preciso para avaliar a disfagia em pessoas idosas ${ }^{56}$. O encaminhamento ao fonoaudiólogo pelo cirurgião-dentista constitui importante avanço no trabalho da equipe interdisciplinar.

Observa-se que há crescente interesse e maiores esclarecimentos entre as evidências acerca de saúde bucal e disfagia, apontando que uma precária condição de saúde bucal está associada à disfagia orofaríngea. Dessarte, é importante que se direcionem maiores esforços para investigações, discussões e estudos sobre variáveis odontológicas e sintomas ligados à dificuldade de deglutição.

\section{Discussão}

A anatomia e a fisiologia da deglutição explicam a associação positiva entre a saúde bucal e a disfagia orofaríngea. Todas as fases da deglutição são essenciais, e cada componente orofacial tem sua função específica. A perda dentária induz mudanças na anatomia da cavidade oral. A capacidade mastigatória depende do número de dentes remanescentes. Assim, a perda dentária perturba a execução coordenada da deglutição. Acredita-se que há um prejuízo na fase oral devido à dificuldade de formação do bolo alimentar, interferindo nas demais fases da deglutição ${ }^{57}$.

$\mathrm{O}$ uso de próteses dentárias desajustadas, especialmente com contato oclusal reduzido, também pode interferir no desempenho mastigatório e dificultar o processo da deglutição. Além disso, as próteses dentárias interferem nos receptores mecânicos e sensoriais do palato duro, resultando em diminuição da estereognosia oral ${ }^{58}$. A formação e a deglutição de um bolo alimentar inadequado em algum momento inoportuno comprometem a integridade do trato respiratório e podem causar disfagia ${ }^{16}$. Assim, o processo do envelhecimento associado a alterações mecânicas pode resultar em alterações na deglutição dos idosos ${ }^{15}$.

No Japão, a quantidade de idosos e o aumento da expectativa de vida repercutiram em avanços nas áreas da deglutição e do envelhecimento. A disfagia é uma disciplina obrigatória no currículo dos profissionais da saúde, e sua carga horária varia entre 30 e 60 horas. Além dos fonoaudiólogos, muitas profissões estão envolvidas na equipe de reabilitação da disfagia, incluindo médicos, cirurgiões-dentistas, enfermeiros, fisioterapeutas, terapeutas ocupacionais, nutricionistas e assistentes sociais. A participação de cirurgiões-dentistas na reabili- tação da disfagia é alta, atuando, principalmente, em reabilitações orais e cuidados com higiene bucal. No entanto, ainda há um pequeno número de configurações de saúde que oportunizam que todas as disciplinas estejam disponíveis para a reabilitação dos pacientes. Os fonoaudiólogos são os principais profissionais que prestam assistência direta aos pacientes disfágicos; entretanto, na sua ausência, há profissionais capacitados para atuação. Assim, modelos de trabalho da equipe transdisciplinar em que as necessidades dos pacientes são tratadas pelo profissional disponível são comuns, garantindo o acesso e o cuidado aos idosos ${ }^{58}$.

A interdisciplinaridade entre Fonoaudiologia e Odontologia é essencial para a integridade do sistema estomatognático, que desempenha as funções orais. Também é importante ressaltar a Fisioterapia como auxílio na terapêutica. Todas essas áreas têm interesses e atuações compartilhados; as atuações estão vinculadas, e o trabalho conjunto oportuniza um melhor prognóstico e o aumento da qualidade de vida dos idosos ${ }^{59}$.

É preciso que profissionais de saúde e gestores atentem para o crescente aumento do número de idosos, sua longevidade e as consequências dessa situação. Ações direcionadas para a atuação interdisciplinar e para a ampliação do acesso da população ao fonoaudiólogo, bem como a profissionais capacitados para tratar a disfagia, devem ser prioridade nos cuidados em saúde dos idosos. Alguns países como o Japão demonstram uma tendência a ser discutida e considerada como exemplo.

\section{Considerações finais}

A falta de cuidados coordenados na atenção ao idoso interfere no seu bem-estar geral e na sua qualidade de vida. O estudo aprofundado com abordagem interdisciplinar do paciente disfágico representa a maneira mais adequada de se atuar nesta área. As evidências da literatura demonstram importante avanço na identificação, no diagnóstico e na reabilitação da disfagia orofaríngea. A reabilitação bucal demonstrou ser relevante para prevenção de alterações na deglutição, e a presença odontológica é crescente nesta área.

\section{Abstract}

The morphological and functional changes that affect the stomatognathic system may be aggravated by tooth loss and the use of dental prosthesis, which compromise mastication and swallowing by the elderly. Objective: thus, the present literature review is proposed, aiming to approach the knowledge that should exist between Speech Therapy and Dentistry, discussing the most significant current, historical, and conceptual evidence on swallowing that bring the two specialties together, especially in elderly individuals. Literature review: a total of 101 studies were included, and they were selected 
from the databases PubMed Central, Scientific Electronic Library Online, Science Direct, SciELO, and Capes journal, identified up to April 2018 in English, Portuguese, and Spanish. Standing out among the findings is the increasing insertion of Dentistry in oropharyngeal dysphagia. The lack of coordinated care for the elderly interferes with their general well-being and quality of life. Final considerations: evidence from the literature shows important advances in the identification, diagnosis, and rehabilitation of oropharyngeal dysphagia, which through oral rehabilitation has been considered significant for preventing swallowing disorders.

Keywords: Aging. Dentistry. Dysphagia. Speech

\section{Referências}

1. Rogers AP. Open-bite cases involving tongue habits. Int J Orthodontia, Oral Surg Radiography 1927; 13(10):837-44.

2. Rix RE. Deglutition and the teeth. Am J Orthodontics and Oral Surg 1946; 32(11):645-9.

3 Straub WJ. The etiology of the perverted swallowing habit. Am J Orthodontics Oral Surg 1951; 37(8):603-10.

4. Marchesan IQ. Fundamentos em fonoaudiologia: aspectos clínicos da motricidade oral. 2. ed. Rio de Janeiro (RJ): Guanabara Koogan; 2005.

5. Garliner D. Myofunctional therapy in dental practice. Miami: Bartel Dental Book; 1971.

6. Petersen PE, Yamamoto T. Improving the oral health of older people: the approach of the WHO Global Oral Health Programme. Com Dent Oral Epidemiol 2005; 33(2):81-92.

7 N'gom PI, Woda A. Influence of impaired mastication on nutrition. Prosth Dent 2002; 87(6):667-73.

8. Joshipura J, Hung HC, Rimm EB, Willett WC, Ascherio A. Periodontal disease, tooth loss, and incidence of Ischemic stroke. Stroke 2003; 34(1):47-52.

9. Glick M, Williams DM, Kleinman DV, Vujicic M, Watt RG, Weyant RJ. A new definition for oral health developed by the FDI World Dental Federation opens the door to a universal definition of oral health. J Am Dent Assoc 2016; 147(12):915-7.

10. Hamdy S, Mikulis DJ, Crawley A, Xue S, Lau H, Henry $\mathrm{S}$, et al. Cortical activation during human volitional swallowing: an event-related fMRI study. Am J Physiol 1999; 277(1):G219-225.

11. Marchesan IQ. Disfagia. In: Marchesan IQ, Bolaffi C, Gomes ICD, Zorzi JL. Tópicos em Fonoaudiologia. v. 2. São Paulo: Lovise; 1995.

12. Schaie KW, Willis SL. Psychometric intelligence and aging. In: Blanchard-fieds F, Hess TM. Perspectives on cognitive change in adulthood and aging. McGraw-Hill College: McGraw Hill; 1996. p. 293-322.

13. Fisberg RM, Marchiani DML, Castro MA, Junior EV, Araújo MC, Bezerra IN, et al. Ingestão inadequada de nutrientes na população de idosos do Brasil: Inquérito Nacional de Alimentação 2008-2009. Rev Saúde Pública 2013; 47(1):222-30.

14. Tanure CMC, Barboza CMP, Amaral JP, Rodrigues MA. A deglutição no processo normal de envelhecimento. Rev CEFAC 2005; 7(2):171-7.

15. Ney DM, Weiss JM, Kind AJ, Robbins J. Senescent swallowing: impact, strategies, and interventions. Nutr Clin Pract 2009; 24(3):395-413.
16. Tulunay-Ugur OE, Eibling D. Geriatric Dysphagia. Clin Geriatr Med 2018; 34(2):183-9.

17. Herzberg EG, Lazarus CL, Steele CM, Molfenter SM. Swallow Event Sequencing: Comparing Healthy Older and Younger Adults. Dysphagia 2018; 33(1):1-9.

18. Tracy JF, Logemann JA, Kahrilas PJ, Jacob P, Kobara M, Krugher C. Preliminary observations of the effects of age on oropharyngeal deglutition. Dysphagia 1989; 4(2):90-4.

19. Fucile S, Wright PM, Chan I, Yee S, Langlais ME. Functional oral-motor skills: do they change with age? Dysphagia 1998; 13(4):195-201.

20. Oncins MC, Freire RMAC, Marchesan IQ. Mastigação: análise pela eletromiografia e eletrognatografia. Seu uso na clínica fonoaudiológica. Dist da Comunicação 2006; 18(2):155-65.

21. Jorge TM, Bassi AKZ, Yarid SD, Silva HM, Silva RPPM, Caldana ML, et al. Relação entre perdas dentárias e queixas de mastigação, deglutição e fala em indivíduos adultos. Rev CEFAC 2009; 11(3):391-7.

22. Ono T, Kumakura I, Arimoto M, Hori K, Dong J, Iwata H, et al. Influence of bite force and tongue pressure on oro-pharyngeal residue in the elderly. Gerodontology 2007; 24(3):143-50

23. Dejaeger E, Pelemans W, Ponette E, Joosten E. Mechanisms involved in postdeglutition retention in the elderly. Dysphagia $1997 ; 12(2): 63-7$.

24. Borges BC, Fulco GM, Souza AJ, Lima KC. Xerostomia and hyposalivation: a preliminary report of their prevalence and associated factors in Brazilian elderly diabetic patients. Oral Health Prev Dent 2010; 8(2):153-8.

25. Thomson WM, Chalmers JM, Spencer JÁ, Slade GD, Carter $\mathrm{KD}$. A longitudinal study of medication exposure and xerostomia among older people. Gerodontology 2006; 23(4):205-13.

26. Thomson WM. Issues in the epidemiological investigation of dry mouth. Gerodontology 2005; 22(6):65-76.

27. Fávaro RAA, Ferreira TNR, Martins WD. Xerostomia: etiologia, diagnóstico e tratamento. Rev Clin Pesq Odontol 2006; 2(4):303-17.

28. Janssen I. Influence of sarcopenia on the development of physical disability: the cardiovascular health study. J Am Geriatr Soc 2006; 54(1):56-62.

29. Sagawa K, Kikutani T, Tamura F, Yoshida M. Factors related to skeletal muscle mass in the frail elderly. Odontology 2017; 105(1):91-5.

30. Yoshida M, Suzuki R, Kikutani T. Nutrition and oral status in elderly people. Jap Dent Science Rev 2014; 50(1):9-14.

31. Agarwal E, Ferguson M, Banks M, Batterham M, Bauer J, Capra S, et al. Malnutrition and poor food intake are associated with prolonged hospital stay, frequent readmissions, and greater in hospital mortality: results from the Nutrition care day survey 2010. Clin Nutr 2013; 32(5):737-45.

32. American speech-language-hearing association (ASHA). Report of the ad hoc committee on dysphagia. ASHA 1978; 29(4):57-8.

33. Holt PR. Management and evaluation of dysphagia. Sppl to Annals of Long-Term Care 2004:2-7.

34. Aviv JE, Sataloff RT, Cohen M, Spitzer J, Ma G, Bhayani $R$, et al. Cost-effectiveness of two types of dysphagia care in head and neck cancer: A preliminary report. Ear Nose Throat J 2001; 80(8):553-8.

35. Martino R, Foley N, Bhogal S, Diamant N, Speechley M, Teasell R. Dysphagia after stroke incidence, diagnosis, and pulmonary complications. Stroke 2005; 36(12):2756-63. 
36. Alagiakrishnan K, Bhanji R, Kurian M. Evaluation and management of oropharyngeal dysphagia in different types of dementia: A systematic review. Arch Gerontol Geriatr 2013; 56(1):1-9.

37. Garcia-Peris P, Parón L, Velasco C, de la Cuerda C, Camblor M, Bretón I. Long-term prevalence of oropharyngeal dysphagia in head and neck cancer patients: Impact on quality of life. Clin Nutr 2007; 26(6):710-7.

38. Rhodus NL, Fricton J, Carlson P, Messner R. Oral symptoms associated with fibromyalgia syndrome. J Rheumatol 2003; 30(8):1841-5.

39. Gilheaney Ó, Zgaga L, Harpur I, Sheaf G, Kiefer L, Béchet S, et al. The Prevalence of Oropharyngeal Dysphagia in Adults Presenting with Temporomandibular Disorders Associated with Rheumatoid Arthritis: A Systematic Review and Meta-analysis. Dysphagi 2017; 32(5):587-600.

40. Hiss SG, Postma GN. Fiberoptic endoscopic evaluation of swallowing. Laryngoscope 2003; 113(8):1386-93.

41. Robbins J, Langmore S, Hind JA, Erlichman M. Dysphagia research in the 21st century and beyond: proceedings from dysphagia experts meeting, August 21, 2001. J Rehabil Res Dev 2002; 39(4):543-8.

42. Marik PE, Kaplan D. Aspiration pneumonia and dysphagia in the elderly. CHEST 2003; 124(1):128-36.

43. Holland G, Jayasekeran V, Pendleton N, Horan M, Jones M, Hamdy S. Prevalence and symptom profiling of oropharyngeal dysphagia in a community dwelling of an elderly population: a self-reporting questionnaire survey. Dis Esophagus 2011; 24(7):476-80.

44. Roy N, Stemple J, Merrill RM, Thomas L. Dysphagia in the elderly: Preliminary evidence of prevalence, risk factors and socioemotional effects. Ann Otol Rhinol Laryngol 2007; 116(11):858-65

45 Clavé P, Verdaguer A, Arreola V. Disfagia orofaríngea en el anciano. Med Clin 2005; 124:742-8

46. Leow LP, Huckabee ML, Anderson T, Beckert L. The impact of dysphagia on quality of life in ageing and Parkinson's disease as measured by the swallowing quality of life (SWAL-QOL) questionnaire. Dysphagia 2010; 25(3):216-20.

47. Ekberg O, Hamdy S, Woisard V, Wuttge-Hannig A, Ortega P. Social and psychological burden of dysphagia: its impact on diagnosis and treatment. Dysphagia 2002; 17(2):139-46.

48. Inoue M. Dysphagia Rehabilitation in Japan. Phys Med Rehabil Clin N Am 2008; 19(4):929-38.

49. Yoneyama T, Yoshida M, Matsui T, Sasaki H. Oral care and pneumonia. Oral care working group. Lancet 1999; 354(9177):515.

50. Ueda K, Yamada Y, Toyosato A, Nomura S, Saitho E. Effects of functional training of dysphagia to prevent pneumonia for patients on tube feeding. Gerodontology 2004; 21(2):108-11.

51. Sagawa K, Kikutani T, Tamura F, Yoshida M. Factors related to skeletal muscle mass in the frail elderly. Odontology 2017; 105(1):91-5.

52. Okabe Y, Takeuchi K, Izumi M, Furruta M, Takeshita T, Shibata Y, et al. Posterior teeth occlusion and dysphagia risk in older nursing home residents: a cross-sectional observational study. J Oral Rehabil 2017; 44(2):89-95.

53 Okamoto N, Morikawa M, Yanagi M, Amano N, Tomioka K, Hazaki K, et al. Association of Tooth Loss With Development of Swallowing Problems in Community-Dwelling Independent Elderly Population: The Fujiwarakyo Study. J Gerontol A Biol Sci Med Sci 2015; 70(12):1548-54.
54. Rech RS, Baumgarten A, Colvara BC, Brochier CW, de Goulart B, Hugo FN, Hilgert JB. Association between oropharyngeal dysphagia, oral functionality, and oral sensorimotor alteration. Oral Dis 2018; 24(4):664-72.

55. Venâncio CPL. Deglutição e envelhecimento. In: Papaléo Netto M. Tratado de Gerontologia. 2. ed. São Paulo: Editora Atheneu; 2007. p. 499-511.

56. Rech RS, Hugo FN, Baumgarten A, Dos Santos KW, de Goulart BNG, Hilgert JB. Development of a simplified dysphagia assessment by dentists in older persons. Community Dent Oral Epidemiol. 2018; 46(3):218-24

57. Mishellany A, Woda A, Labas R, Peyron MA. The challenge of mastication: preparing a bolus suitable for deglutition. Dysphagia 2006; 21:87-94.

58. González-Fernández M, Huckabee ML, Doeltgen SH, Inamoto Y, Kagaya H, Saitoh E. Dysphagia Rehabilitation: Similarities and Differences in Three Areas of the World. Curr Phys Med Rehabil Rep 2013; (4):296-306.

59. Garcia MAA, Pinto ATBCS, Odoni APC, Longhi BS, Machado LI, Linek MDS, et al. Interdiscipliaridade e integralidade no ensino em saúde. Rev Ciênc Méd 2006; 15(6):473-85.

\section{Endereço para correspondência:}

Juliana Balbinot Hilgert

Faculdade de Odontologia, Universidade Federal do

Rio Grande do Sul

Rua Ramiro Barcelos, 2492, Santa Cecília.

90035-003, Porto Alegre, Rio Grande do Sul, Brasil

Telefone: (+55) (51) 3308-5204

E-mail: jhilgert@gmail.com

Recebido: 25/01/18. Aceito: 21/03/18 